\title{
Multidimensional energy barrier distributions of interacting magnetic particles evaluated at different magnetization states
}

\author{
O. Chubykalo-Fesenko ${ }^{\text {a) }}$ \\ Instituto de Ciencia de Materiales de Madrid, CSIC, Cantoblanco 28049, Madrid \\ R. W. Chantrell ${ }^{\text {b) }}$ \\ Physics Department, York University, York, YO10 5DD, United Kingdom
}

(Presented on 10 November 2004; published online 12 May 2005)

\begin{abstract}
We evaluate the energy barrier distributions of coupled Co particles as a function of their concentration, which changes the strength of magnetostatic interactions, in a multidimensional space to take into account possible collective reversal. The distributions are evaluated at the remanence, demagnetized state and coercivity for Co particles with 2D random anisotropy easy axes orientations and with concentrations up to $56 \%$. We show that the overall influence of interactions in this system is always to broad the energy barrier distribution. Consequently, in all the cases the magnetization decay would start earlier when the strength of interactions increases. At the remanence, strong interactions displace the average energy barrier to larger values. However, at the coercive force it is displaced to smaller values. At the same time, at the demagnetized state, the peak of the distribution practically remains at the same position. () 2005 American Institute of Physics. [DOI: $10.1063 / 1.1853834]$
\end{abstract}

Many experimental and theoretical studies have been devoted to the problem of long-time temperature induced magnetization decay in nanostructured and particulate magnetic systems. The importance of the problem is also related to the challenge of thermal stability of magnetic recording information. ${ }^{1}$ The specific features of thermal decay of each magnetic system arise from the particle volume and anisotropy distributions and magnetic interactions which influence the energy barrier distribution. It is common to characterize experimentally the thermal behavior of a magnetic media by the effective energy barrier distribution. The experimental methods involve the ac-susceptibility measurements, the use of zero-field-cooled and field-cooled dc susceptibility curves measured at low magnetic fields or dynamic remanent hysteresis loops. This experimental characterization of thermal effects of a magnetic sample is based on the concept of timeindependent energy barrier distribution in systems with weak interactions. However, generally speaking, in an interacting system the energy barrier distribution is a dynamical (time and field dependent) property. The interpretation of the "effective" energy barrier distribution extracted from experimental data is, therefore, often not straightforward.

The influence of interactions on the energy barrier distribution has been a subject of many investigations. There is no unique opinion on the question and many contradictory results may be found in the literature. For example, Luis et $a .^{2}$ have compared the effective energy barriers obtained from ac susceptibility for single and multilayered Co particle samples and concluded that the magnetostatic interactions displace the effective energy barriers to larger values. On the contrary, Morup and Tronc argued that the blocking temperature may be decreased due to dipolar interactions. ${ }^{3}$ From this point of view, it is necessary to have the capability of theo-

\footnotetext{
${ }^{a)}$ Electronic mail: oksana@icmm.csic.es

${ }^{b)}$ Electronic mail: rc502@york.ac.uk
}

retical predictions in some simple modelling situations.

Most theoretical models which deal with the energy barriers use the local field approximation. The interactions are included in the energy barrier as an effective field acting on each particle. This field may be evaluated on the basis of numerical calculation of the interaction field acting on a particular particle. ${ }^{4}$ However, none of these methods are capable of predicting the energy barriers associated with thermal collective magnetisation reversal over long timescales. These involve energy barrier calculations in a multidimensional energy space. Recently, several authors ${ }^{5,6}$ have overcome the one particle approximation and calculated the energy barrier distributions in a multidimensional space. In this paper we use an extension of previous methods to develop a model of energy barriers in systems of interacting particles. The model is used to study the effects of magnetostatic interactions in systems with varying packing densities. It is found that there is a strong dependence of the energy barrier distribution on the magnetic state and on the physical microstructure of the system.

In this paper we calculate the energy barrier distributions in a multidimensional space. Initially, to approximate the saddle point we use the ridge optimization method described in Ref. 7. This method involves firstly searching for a maximum in a given direction. This determines a point on the curve separating two local minima. The method then brackets the maximum and searches down thesteepest gradient for the lowest maximum (saddle point). However, this method converges only slowly to the saddle point. To converge to the saddle point with a given precision, we use the augmented Hessian method. ${ }^{8}$ Finally, normal mode analysis is performed to assure that the negative eigenvalue is unique. This check is necessary to ensure that the point obtained is not a higher-order saddle which may artificially couple several independent energy barriers. Finally, we have found it necessary to check that the obtained saddle point separates the 

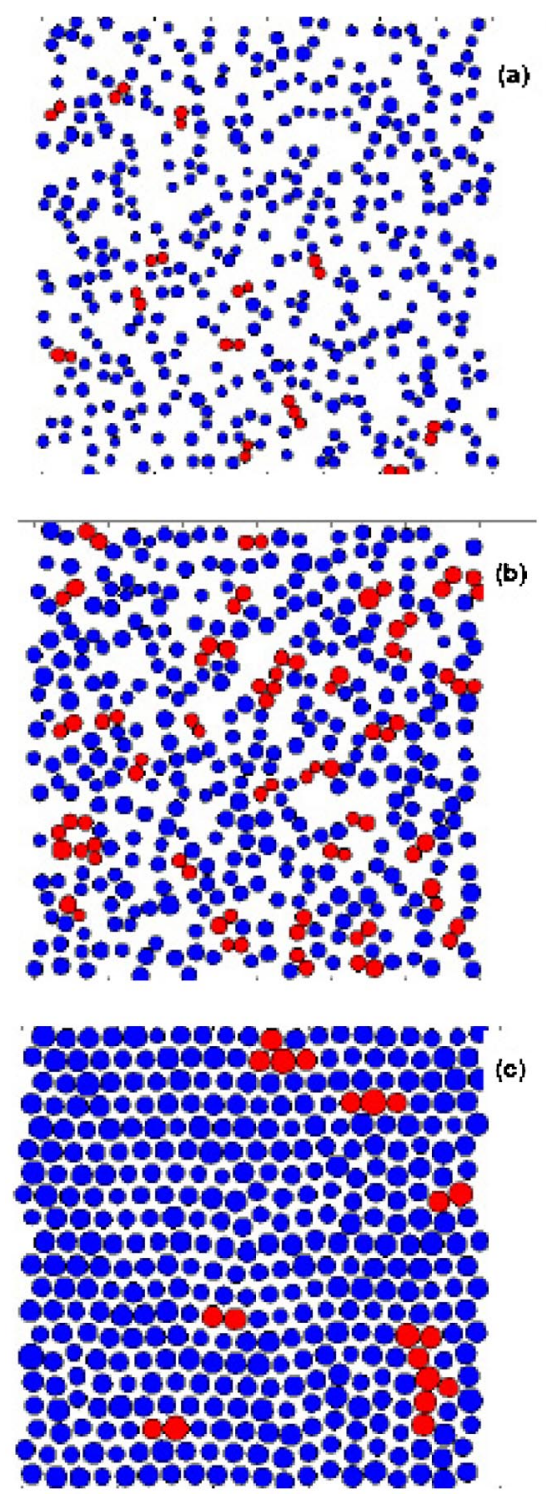

FIG. 1. (Color online) Generated particle distributions with $\sigma=0.1$ and different concentrations: (a) $c=0.2$, (b) $c=0.36$, (c) $c=0.56$. The particles marked by a bright color touch each other and are considered to be exchange coupled.

basin of attraction of two minima, of which one is the initial state. The total numerical scheme is computationally costly due to the fact that in a complex energy landscape of an interacting magnetic system the ridge has also a complex character, in particular, it may curve, bifurcate and disappear. In the case of Co particles which have a large anisotropy constant, we have found that the best choice for the initial trajectory guess is an individual rotation of each particle in both directions in the film plane.

In the present paper we consider realistic 2D arrays of 400 Co particles with log-normal size distribution with the most probable particle diameter $4 \mathrm{~nm}$ and $\sigma=0.1$. The anisotropy value is taken to be $K=2 \cdot 10^{6} \mathrm{erg} / \mathrm{cm}^{3}$, the magnetization saturation value $M_{s}=1400 \mathrm{emu} / \mathrm{cm}^{3}$ and a $2 \mathrm{D}$ random anisotropy easy axes distribution was supposed. To generate realistic particle arrangements we use a specially designed algorithm as described in detail in Ref. 9. Essentially, an initial state is produced under the influence of a strong repul-

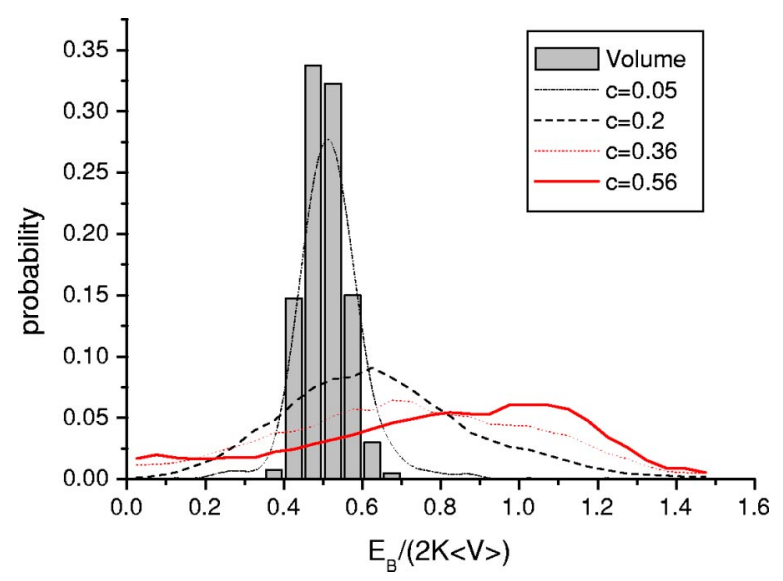

FIG. 2. Energy barrier distributions for coupled Co particles with different concentrations at the remanent state.

sive potential, which shows some degree of self-assembly as, for example, in Fig. 1(c). The particles are then allowed to move under the influence of an attractive central potential, which leads to structures with short-ranged order dependent upon the packing density, as shown in Figs. 1(a) and 1(b). As can be seen in Fig. 1, the algorithm produces the formation of chains and loops of particles for intermediate concentrations and that of the self-organized quasicrystal hexagonal structure for large particles concentration. In this paper we are most concerned with the effects of the magnetostatic interactions in systems with varying degrees of order as shown in Fig. 1.

The remanent state was prepared starting with large saturating field and slowly decreasing its value to zero, minimizing the total energy at each time step. The energy barrier distributions in this state are presented in Fig. 2 for different particle concentrations. The overall effect of the magnetostatic interactions is to broaden the distribution and to displace its center to larger values. We have observed the splitting of the original volume distribution into two subdistributions with low and high energy barriers for each particle. In the presence of interactions the two energy barriers of an individual particle (corresponding to two senses of rotation) behave differently. While the height of the larger barrier increases with the strength of interaction, the behavior of the smaller barriers depends on the invironment; in particular it may decrease or disappear. We have also observed a change in the form of distributions for large concentrations from having tails to high energies (characteristic of log-normal distributions) to having tail to lower energies. However, the total amount of small energy barriers is always increased with increasing magnetostatic strength. Consequently, despite the fact that the distribution center is displaced to larger values, this will not lead to additional thermal stability of the corresponding media. As may be expected, the average cluster size of particles participating in the collective reversal is increased with the strength of magnetostatic interactions up to 6 in this case.

Secondly, we have calculated the energy barrier distribution at the coercivity as a function of particle concentration (Fig. 3). As might be expected, at the coercivity the amount of small barriers is considerably increased. In the absence of 


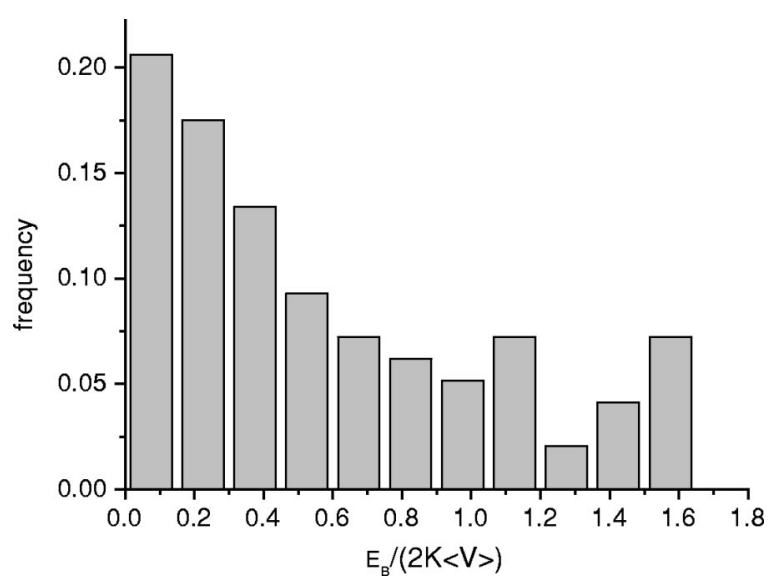

FIG. 3. Energy barrier distribution of magnetostatically coupled Co particles with $c=0.2$ concentration at the coercivity.

interactions, a two-peak distribution, corresponding to switching towards the parallel and antiparallel field directions has been predicted in Ref. 10. This is due to progressive splitting of the original energy barrier distribution into two subdistributions with low and high energy barriers as external field increases for the case of noninteracting particles. This effect is apparently smoothed by magnetostatic interactions.

It is also common to investigate the energy barrier distribution at the ac-demagnetized state. To simulate the demagnetized state, we minimized the energy of the system with random initial conditions achieving the magnetization of less than 0.01. It should be noted that this process produces a magnetic state which may differ from that produced by an experimental ac-demagnetization procedure, which is carried out by applying a sequence of hysteresis cycles in gradually decreasing fields. Figure 4 presents the energy barrier distribution for the Co particle at the demagnetized state for two concentrations. In comparison with the corresponding graph in Fig. 2, the distribution has the same width but its peak is still located around $K\langle V\rangle$ value, i.e., no significant displacement of the distribution center is observed.

In conclusion, the energy barrier distributions depend significantly on the magnetization state. We have observed

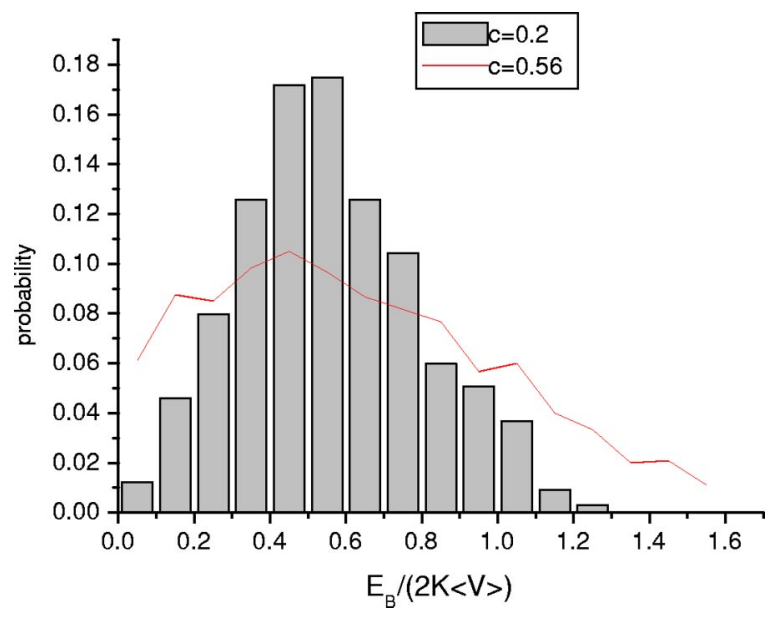

FIG. 4. Energy barrier distribution of magnetostatically coupled Co particles with two concentrations at the demagnetized state. that the magnetic interactions always broaden the energy barrier distribution. There is no unique energy barrier distribution with which it is reasonable to characterize the magnetic material. The form of the distribution changes dynamically with time during the field or temperature induced magnetization decay. Although at remanence we have observed the displacement of the energy barrier distribution center to larger values, this displacement may be irrelevant for the rate of magnetization decay where only small barriers participate.

Our calculations in some sense agree with existing experimental observations: most experimental measurements have observed an increase of blocking temperature of superparamagnetic particles when the average distance between particles decreases. ${ }^{11}$ However, the relaxation of more concentrated systems of particles have been often measured to be faster. ${ }^{3}$ This latter observation is consistent with our calculations which predict the increment of the amount of small barriers (responsible for faster decay) and displacement of the distribution center for larger values. Previous studies using MC models (without collective reversal) ${ }^{12,13}$ have also noted this effect. In particular in Ref. 12 it was shown that in 3D case the blocking temperature increased with interaction strength at the demagnetized state. In Ref. 13, the conflicting experiments were also discussed and it was shown that interactions can increase the energy barrier in interacting systems resulting in hysteretic behavior in systems exhibiting superparamagnetic behavior in the noninteracting state. We should also point out that our results are in contradiction with the preliminary results of Berkov, ${ }^{5}$ who calculated the energy barrier distributions in a multidimensional space for particulate systems with varying degrees of magnetostatic interactions in the absence of external field. He concluded that the magnetostatic interactions displace the energy barrier distributions to smaller values which differs from the conclusions of our work. However, it is important to recall that the actual energy barrier distribution depends on the detail of the magnetic state. It is likely that a major contribution to this discrepancy lies in the preparation of the demagnetized state (not specified in Ref. 5). Further work is necessary in order to test this hypothesis.

\footnotetext{
${ }^{1}$ A. Moser and D. Weller, in The Physics of Ultra-High-Density Magnetic Recording (Springer, New York, 2001), p. 145.

${ }^{2}$ F. Luis, J. M. Torres, L. M. Gracía, J. Bartolomé, J. Stankiewicz, F. Petroff, F. Fettar, J.-L. Maurice, and A. Vaurès, Phys. Rev. B 65, 094409 (2002).

${ }^{3}$ S. Mørup and E. Tronc, Phys. Rev. Lett. 72, 3278 (1974).

${ }^{4}$ C. Verdes, B. Ruiz-Diaz, S. M. Thompson, R. W. Chantrell, and Al. Stancu, Phys. Rev. B 65, 174417 (2002).

${ }^{5}$ D. V. Berkov, J. Appl. Phys. 83, 7390 (1998); J. Magn. Magn. Mater. 186, 199 (1998).

${ }^{6}$ O. Chubykalo and R. W. Chantrell, J. Magn. Magn. Mater. 272-276, E1169 (2004).

${ }^{7}$ A. Lyberatos and R. W. Chantrell, Phys. Rev. B 52, 4301 (1995).

${ }^{8}$ B. Lengsfield, J. Chem. Phys. 73, 382 (1980).

${ }^{9}$ K. Yu. Guslienko, T. J. Klemmer, X. W. Wu, R. W. Chantrell, D. Weller, and O. Chubykalo, Phys. Rev. B (submitted).

${ }^{10}$ O. Iglesias, A. Labarta, Comput. Mater. Sci. 25, 577 (2002).

${ }^{11}$ S. Morup et al., J. Magn. Magn. Mater. 40, 163 (1983); M. El-Hilo, K. O'Grady, and R. W. Chantrell, ibid. 117, 21 (1992).

${ }^{12}$ R. W. Chantrell, N. S. Walmsley, J. Gore, and M. Maylin, Phys. Rev. B 63, 24410-14 (2001).

${ }^{13}$ D. Kechrakos and K. N. Trohidou, Phys. Rev. B 58, 12169 (1998).
} 